\title{
Patients' Willingness to Accept the Risks and Benefits of New Treatments for Chronic Hepatitis C Virus Infection
}

\author{
Teresa L. Kauf, ${ }^{1}$ Ateesha F. Mohamed, ${ }^{2}$ A. Brett Hauber, ${ }^{2}$ Derek Fetzer ${ }^{3}$ and Atiya Ahmad \\ 1 Department of Pharmaceutical Outcomes and Policy, College of Pharmacy, University of Florida, \\ Gainesville, FL, USA \\ 2 RTI Health Solutions (RTI-HS), Research Triangle Park, NC, USA \\ 3 Johnson \& Johnson Pharmaceutical Services, Raritan, NJ, USA
}

\begin{abstract}
Background: Some patients with chronic hepatitis $\mathrm{C}$ virus (HCV) infection forego treatment due to concerns about treatment efficacy, treatment duration, and side effects.

Objective: The purpose of this study was to quantify patient preferences among possible outcomes associated with new, direct-acting antiviral agents (DAAs) for the treatment of $\mathrm{HCV}$ infection and determine which treatment features are most important to patients in making treatment decisions.

Methods: Adult participants with a self-reported physician diagnosis of $\mathrm{HCV}$ infection in five countries completed a web-enabled, choice-format conjoint analysis survey. The survey presented participants with 20 treatment-choice questions. Each treatment-choice question included a pair of hypothetical treatment profiles with varying levels of six attributes: treatment duration; chance of getting rid of the virus completely (i.e. likelihood of a sustained virologic response [SVR]); weeks on an additional, third medicine (i.e. a DAA); risk of a severe rash; risk of severe anemia; and number of times a day the third medicine is taken. Treatment-choice questions were based on a predetermined experimental design with known statistical properties. Randomparameters logit was used to estimate preference weights for all attribute levels and the mean relative importance of each attribute.

Results: 284 participants completed the survey. Likelihood of an SVR was the most important outcome to participants, followed by severe anemia risk, severe rash risk, therapy type (a combination of total weeks of treatment and weeks on the third medicine), and dosing of the third medicine. Controlling for other factors, preferences were similar across all therapy types examined. Conclusion: Patients with HCV infection indicate a willingness to accept an increased risk of side effects for sufficient improvement in the likelihood of treatment response.
\end{abstract}




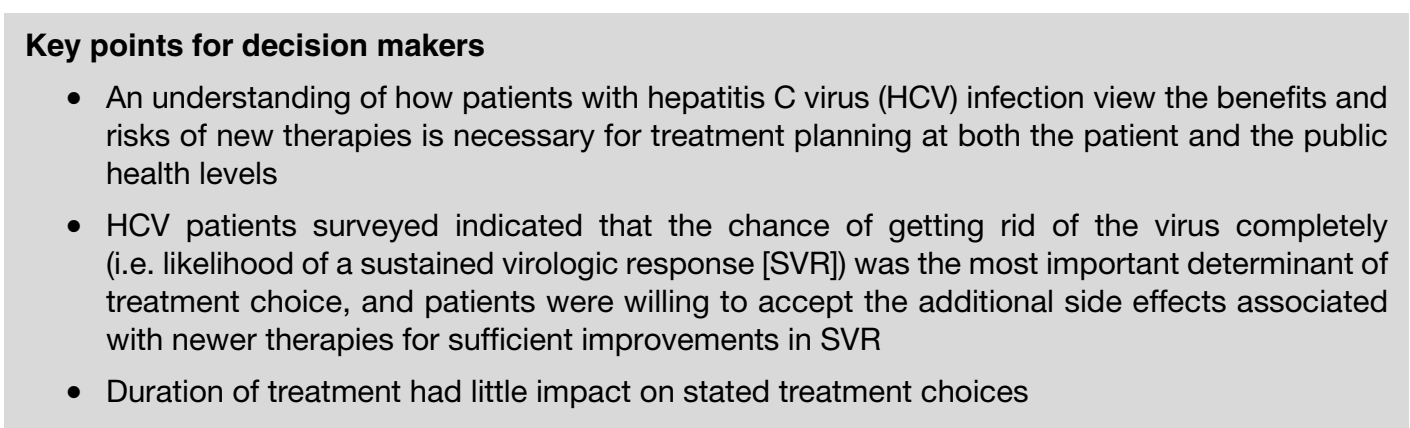

\section{Introduction}

Chronic hepatitis $\mathrm{C}$ virus ( $\mathrm{HCV}$ ) infection damages the liver and, over time, can cause cirrhosis, liver failure, and liver cancer. Not all patients with $\mathrm{HCV}$ infection will experience these severe manifestations, ${ }^{[1]}$ but the majority of patients will exhibit some degree of liver damage upon diagnosis due to the slow, asymptomatic progression of the disease. ${ }^{2]}$ Treatment generally is recommended for patients with significant liver fibrosis and often offered to patients in earlier stages of liver disease.

For a number of years, the standard of care for the treatment of chronic HCV infection has been combination therapy with pegylated interferon (peginterferon) plus ribavirin. ${ }^{[2,3]}$ For patients infected with genotype 1 virus, combination antiviral therapy is effective in producing a sustained virologic response (SVR) in about half of those initiating treatment. Rates of SVR have improved over time, as therapy has evolved from monotherapy and combinations that included the non-pegylated form of interferon. ${ }^{[2]}$ Despite improvements in treatment effectiveness, the number of patients seeking therapy for chronic HCV infection has not increased over time, and evidence suggests that only a small percentage of patients - sometimes as few as $6 \%$ - receive antiviral therapy. ${ }^{[4-6]}$

Commonly cited reasons for low rates of treatment among chronically infected individuals include contraindications to therapy, ${ }^{[5,7]}$ the slowly progressive nature of the disease, ${ }^{[8,9]}$ low treatment efficacy, ${ }^{[8,9]}$ and treatment burden including treatment duration, ${ }^{[5]}$ side effects, ${ }^{[8,9]}$ and cost. ${ }^{[8]}$ Treatment with new and emerging, direct-acting antiviral agents (DAAs) for HCV may offer im- proved rates of SVR (and, for some patients, reduced treatment duration), but they are not without additional side effects, cost, and other potential complications, such as the development of drug resistance. ${ }^{[10]}$ Furthermore, these new treatments are administered alongside peginterferon and ribavirin, potentially compounding patients' concerns about the burden of treatment.

While some evidence of patient preferences regarding $\mathrm{HCV}$ treatments exists, ${ }^{[1,12]}$ the available literature focuses on treatment with ribavirin plus interferon or peginterferon. In this paper, we report the results of a choice-format conjoint analysis survey that asked patients to choose among alternative, hypothetical HCV treatments that include characteristics of new DAAs. Each hypothetical treatment in the survey varied by treatment effectiveness, duration, risk of side effects, and additional pill burden associated with DAAs.

Choice-format conjoint analysis is a systematic method of eliciting trade-offs to quantify the relative importance participants assign to various treatment attributes or outcomes. It is based on the premise that medical interventions are composed of a set of attributes or outcomes and that the attractiveness of a particular intervention to an individual is a function of these attributes. ${ }^{[13,14]}$ Conjoint techniques are grounded in both psychology and economic theory ${ }^{[15,16]}$ and offer several advantages over simple rating or ranking exercises, including the ability to examine the strength of individual preferences across treatment attributes and observe the willingness to trade between different attributes. ${ }^{[14,17]}$ While the treatment combinations presented in our survey are hypothetical in nature, 
the specific features of the treatments reflect the characteristics of new DAAs that are of most importance to patients.

\section{Methods}

\section{Survey Instrument}

A choice-format conjoint survey instrument was constructed to reflect key aspects of $\mathrm{HCV}$ treatment with the current standard of care, with or without DAAs (see the Supplemental Digital Content (SDC), http://links.adisonline.com/PBZ/ A40). Standard of care was described as an injection with a pen once a week (peginterferon) and a tablet twice a day (ribavirin) for 48 weeks; ${ }^{[2,3]}$ the third drug representing the DAA was assumed to be administered orally. Relevant treatment features and levels were chosen based on an informal review of relevant literature (including package inserts and online sources of information about HCV treatment), consultation with clinical experts, the authors' previous experience conduct- ing choice studies in $\mathrm{HCV}$, and face-to-face interviews with $\mathrm{HCV}$-infected patients. Six treatment attributes were determined from this process (table I): total number of weeks on treatment; chance of getting rid of the virus completely (i.e. chance of an SVR, defined as the absence of plasma HCV ribonucleic acid [RNA] 6 months after therapy completion ${ }^{[2,3]}$ ); number of weeks the third medicine is taken; chance of a treatmentlimiting severe rash; chance of severe anemia; and the number of times a day the third medicine is taken.

A number and range of levels were assigned to each treatment attribute. Greater numbers of levels allow for the estimation of non-linear preferences within attributes. However, because the sample size requirements increase exponentially with the number of levels included for each attribute, the number of levels generally was limited to three with the exception of the duration attributes 'total number of weeks on treatment' and 'number of weeks the third medicine is taken.' The duration attributes were combined to create

Table I. Attributes and levels for the treatment-choice questions

\begin{tabular}{|c|c|c|}
\hline Attribute labels & Abbreviated label & Levels \\
\hline Total number of weeks on treatment ${ }^{a}$ & Total weeks & $\begin{array}{l}24 \text { weeks } \\
28 \text { weeks } \\
48 \text { weeks }\end{array}$ \\
\hline $\begin{array}{l}\text { Chance that the treatment will get rid of the } \\
\text { virus completely }\end{array}$ & Chance of getting rid of virus completely & $\begin{array}{l}85 \text { out of } 100(85 \%) \\
70 \text { out of } 100(70 \%) \\
45 \text { out of } 100(45 \%)\end{array}$ \\
\hline Number of weeks the third medicine is taken ${ }^{a}$ & No. weeks on 3rd medicine & $\begin{array}{l}0 \text { weeks (no third medicine) } \\
12 \text { weeks } \\
24 \text { weeks (with 4-week lead-in) } \\
44 \text { weeks (with 4-week lead-in) }\end{array}$ \\
\hline $\begin{array}{l}\text { Chance of getting a severe rash that requires } \\
\text { stopping the third medicine }\end{array}$ & Chance of severe rash & $\begin{array}{l}\text { No chance of severe rash }(0 \%)^{b} \\
5 \text { out of } 100(5 \%) \\
12 \text { out of } 100(12 \%)\end{array}$ \\
\hline $\begin{array}{l}\text { Chance of getting severe anemia that requires } \\
\text { additional injections of a fourth medicine while } \\
\text { taking the third medicine }\end{array}$ & Chance of severe anemia & $\begin{array}{l}\text { No chance of severe anemia }(0 \%)^{\mathrm{b}} \\
20 \text { out of } 100(20 \%) \\
70 \text { out of } 100(70 \%)\end{array}$ \\
\hline Number of times a day the third medicine is taken & No. times/day the 3rd medicine is taken & $\begin{array}{l}\text { No additional tablet }{ }^{\mathrm{b}} \\
3 \text { tablets every } 12 \text { hours ( } 2 \text { times/day) } \\
2 \text { tablets every } 8 \text { hours ( } 3 \text { times/day) } \\
4 \text { tablets } 3 \text { times a day }\end{array}$ \\
\hline \multicolumn{3}{|c|}{$\begin{array}{l}\text { The levels of the 'total number of weeks on treatment' and 'number of weeks the third medicine is taken' attributes were constrained to } \\
\text { reflect five hypothetical treatments of interest, as shown in table II. }\end{array}$} \\
\hline b These attribute levels occur together whenever & treatment does not include a third drug. & \\
\hline
\end{tabular}


Table II. Hypothetical treatments of interest

\begin{tabular}{lll}
\hline Treatment & Total number of weeks on treatment ${ }^{\mathrm{a}}$ & Number of weeks the third medicine is taken \\
\hline A & 24 weeks & 12 weeks \\
B & 28 weeks & 12 weeks \\
C (standard of care) & 48 weeks & 0 weeks (no third medicine) \\
D & 48 weeks & 24 weeks (with 4-week standard-of-care lead-in) \\
E & 48 weeks & 44 weeks (with 4-week standard-of-care lead-in) \\
\hline a & Pegylated interferon plus ribavirin are taken for the entire duration of treatment. & \\
\hline
\end{tabular}

five therapy options reflecting both the current standard of care and the probable treatment patterns with DAAs (table II). The levels assigned to dosing frequency and SVR chance similarly were chosen to represent realistic treatment options. Upper ranges of probabilities for the side effect attributes were roughly based on clinical trial reports but also designed to be large enough to induce respondents to refuse an otherwise acceptable treatment profile. Levels for the side effect and dosing attributes were set to 'no chance ...' and 'no additional tablet,' respectively, when the treatment option presented reflected the current standard of care (see, for example, treatment A in figure 1).

\section{Task Construction and Experimental Design}

Attributes and levels were combined to create pairs of hypothetical treatment profiles. Survey participants were asked to indicate which hypothetical treatment in each pair they would choose if the two treatments shown were the only options available. An example treatment-choice question is shown in figure 1. Participants answered 20 treatment-choice questions (figure 1) from among the constructed treatment profiles.

To create treatment profiles for the choice questions, we employed a commonly used Defficiency algorithm. ${ }^{[18-20]}$ Side effect chances were constrained to zero when no third medicine was included in a choice task. This reduces the orthogonality and efficiency of the design but is necessary to allow estimation of preferences across treatments, independent of the other attributes in the survey. This process produced a statistically efficient experimental design resulting in 72 choice pairs, eight survey versions with nine treatmentchoice questions each. The order of the nine ques- tions was randomized, and the choice pair appearing in question three was repeated as question ten as an internal validity test. The final experimental design consisted of eight survey versions, each containing ten treatment-choice questions. Each participant was randomly assigned to two of the eight versions.

\section{Instrument Design and Pre-Testing}

Prior to answering the treatment-choice questions, participants viewed descriptions of the attributes and levels, including a graphic tutorial to communicate risk. ${ }^{[21]}$ Participants were reminded that all treatments included peginterferon plus ribavirin and told to assume that any side effects of these drugs, such as flu-like symptoms, fatigue, and depression, were the same for each treatment. Participants were informed that any side effects shown in the treatment-choice questions were only for the third drug and would resolve upon discontinuation. Participants also were asked to assume that all medical bills, including the cost of medicines, were covered by health insurance. Standard demographic information (e.g. age, gender, marital status, and education) and questions about participants' experiences with HCV infection and treatment were included in the survey.

The survey was pre-tested through a series of one-on-one, face-to-face interviews conducted in the UK and the US. During the interviews, participants were asked to read the survey out loud and think aloud as they answered the survey questions. Two members of the study team recorded answers to all questions and noted any difficulties understanding any of the information presented, including the choice tasks. Additionally, the study team queried participants to understand how the attributes and levels affected 
participants' decision making. This process was used to identify attributes that did not influence treatment choice (e.g. risk of jaundice) and confirm ranges for the levels of probabilistic attributes, as described in the Survey Instrument section. The pre-test sample in the UK consisted of eight individuals who self-reported current or previous treatment for chronic HCV infection. The US sample $(n=7)$ included both treatment-naïve and treatment-experienced patients of the Adult Liver Clinic at the University of Florida (UF).

The study was approved by the UF Institutional Review Board (Gainesville, FL, USA) and Research Triangle Institute (RTI) International's Office of Research Protection and Ethics (Research Triangle Park, NC, USA). All survey participants were required to provide informed consent.

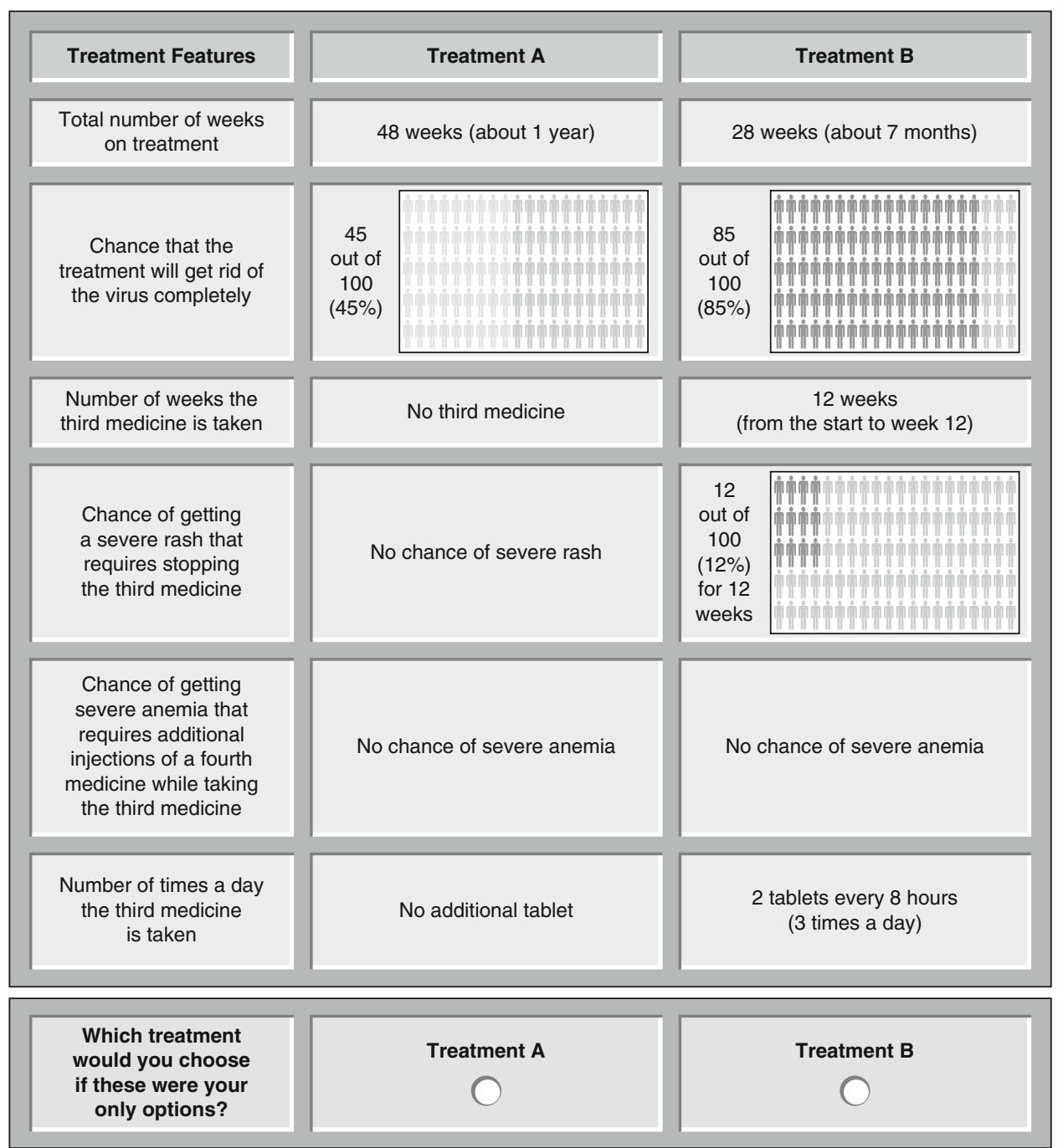

Fig. 1. An example treatment-choice question. In the survey, different colors were used to represent different likelihoods of chance events (see the Supplemental Digital Content, http://links.adisonline.com/PBZ/A40, for further information). 


\section{Data Collection}

Survey participants were required to have a self-reported physician diagnosis of HCV infection and be at least 18 years of age. Knowledge Networks (KN), a company that specializes in survey research using online surveys, recruited participants in five countries - the UK, France, Germany, Spain, and the US - from two well established online patient panels. Panel members 'opt in' to the panels and agree to participate in online surveys without remuneration.

Email invitations were sent to panel members who previously reported a diagnosis of HCV infection in a comprehensive health condition questionnaire. Panel members who had not completed the health condition questionnaire were invited and screened for a self-reported physician diagnosis of $\mathrm{HCV}$ infection. Potential participants from the general population enrolled in other $\mathrm{KN}$ consumer panels were screened to complete the sample. KN administered the web-enabled survey in November 2010.

\section{Statistical Analysis}

Summary statistics were calculated for demographic, disease, and treatment history variables for all participants. An internal stability test as described in the Task Construction and Experimental Design section was conducted for all respondents. Multivariate analyses were conducted on samples excluding and including participants who 'failed' the stability test. Respondent choices also were examined for dominance. For the purposes of this study, dominance was defined as choosing the treatment option with the better level of a specific attribute on at least 15 of the 20 choice questions. Because some individuals may have very strong preferences over a specific treatment attribute, there was no plan to exclude those who dominated from the multivariate analysis. ${ }^{[22]}$ However, individuals who showed no variation in their responses - that is, they always chose treatment $\mathrm{A}$ or treatment $\mathrm{B}$ - were excluded.

Multivariate random-parameters logit was used to estimate the effect of each treatment attribute on the probability of choosing a treat- ment. Random-parameters logit is a limited dependent-variable estimator in which the dependent variable is treatment choice and the independent variables are the levels of each attribute included in the survey. This estimation method controls for within-participant correlation and avoids potential estimation bias from unobserved preference weight heterogeneity by estimating a distribution of preference weights across participants for each treatment attribute. ${ }^{[23,24]}$ Heterogeneity of preferences within attribute levels was assessed using t-statistics.

The parameter estimates from the logit model can be interpreted as mean preference weights or as log-odds ratios, indicating the relative strength of preference for each attribute level. ${ }^{[25]}$ The distance between parameter estimates for the best and worst levels of an attribute can be interpreted as the overall relative importance of the attribute over the specific ranges presented in the survey. For example, the importance of SVR was estimated as the importance of increasing chance of SVR from $45 \%$ to $85 \%$. Relative importance scores are calculated by assigning a value of ten to the most important attribute and scaling the importance weights for the remaining attributes comparatively.

The relative importance scores are a subset of the preference weights. The preference weights, calculated as log-odds ratios from the parameter estimates, indicate the strength of preference for each attribute level. The distance between preference weights for the best and worst level within an attribute yields the relative importance of that attribute overall. We report both derivations because the preference weights provide information about the attributes and the levels. The relative importance scores only provide information about each attribute as a whole (again, over the range of levels examined in the survey).

All treatment attributes were modeled as random, effects-coded categorical variables with Normal distributions. ${ }^{[13,26]}$ A dummy variable was used to represent standard of care, which always appeared in the choice tasks with no chance of severe rash or severe anemia. The other four therapies shown in table II were modeled as a categorical variable. Therefore, the results are interpreted as the relative importance of each therapy and treatment 
attribute, controlling for standard of care as defined above.

\section{Results}

\section{Study Sample}

Email invitations were sent to 4936 respondents with self-reported HCV-infection (UK = 483; France $=1729$; Germany $=655$; Spain $=424$; $\mathrm{US}=1645)$. Initial response rates across the five countries varied from $10 \%$ to $47 \%(\mathrm{UK}=35 \%$; France $=37 \%$; Germany $=38 \%$; Spain $=47 \%$; US $=$ $10 \%)$. Among those initially responding to the email invitation, 284 participants met the inclusion criteria and completed the survey (UK = 56; France $=56$; Germany $=58 ;$ Spain $=58 ; \mathrm{US}=56$ ). The majority of participants were male $(53 \%)$, employed (63\%), and had at least a 4-year college education $(53 \%)$. Forty-nine percent of participants were married, and $26 \%$ were single. The mean age of the participants was 42 (standard deviation [SD] 12) years.

Participants' disease and treatment experiences and other characteristics are summarized in table III. All participants except those residing in Spain answered the disease and treatment experience questions. The majority of the remaining participants $(62 \%)$ were diagnosed with $\mathrm{HCV}$ infection 2 or more years prior to completing the survey. With respect to treatment experience, approximately $27 \%$ were treatment naïve, $21 \%$ were on treatment for the first time, $33 \%$ were not currently on treatment but had been treated in the past, and $20 \%$ were currently on treatment and also had been treated in the past. Among participants who were untreated or waited before seeking treatment, most reported delayed treatment because they did not realize that hepatitis was serious, their doctors told them that treatment was not yet required, or they were worried about the side effects of treatment.

Fewer than $3 \%$ of participants dominated on a particular treatment attribute: one dominated on SVR, one on the number of weeks the third medicine was taken, two each on risk of severe anemia and severe rash, and one on the number of times per day the third medicine was taken.
These seven individuals' observations were included in the regression analysis. Eight participants who selected the same treatment option across all the treatment-choice questions presented were excluded from the sample. The final sample size used to estimate HCV treatment preferences was 276. Results from the random-parameters logit regression model and the SDs for each parameter distribution are given in table IV. These results include data from 79 respondents who failed the stability test; model results with and without these data were not statistically different. Results also did not differ between treatment-naïve and -experienced patients.

\section{Preference Weights}

Figure 2 shows the preference weight log-odds ratios relative to the mean attribute effect normalized at zero. The vertical bars around each parameter indicate the $95 \%$ confidence interval (CI) for the estimate. The estimated preference weights for the effects-coded attributes were consistent with the natural ordering of the categories; that is, better clinical outcomes were preferred to worse clinical outcomes.

The vertical distance between adjacent preference weights indicates the relative importance of moving from one level of an attribute to an adjacent level of that attribute. For example, the relative importance of an improvement in the chance of severe rash from $5 \%$ to $0 \%$ is approximately 0.24 . Similarly, the relative importance of an improvement in the chance of SVR from $70 \%$ to $85 \%$ is approximately 0.77 . Therefore, the importance of an improvement from $70 \%$ to $85 \%$ chance of SVR is 3.1 times as important as an improvement from $5 \%$ to $0 \%$ in the chance of severe rash.

\section{Relative Importance Scores}

The distance between preference weights for the best and worst levels of an attribute can be interpreted as the overall relative importance of the attribute over the specific ranges presented in the survey. Figure 3 presents the mean relative importance scores (and 95\% CIs) for the attributes included in the survey. The absolute scale of the relative importance scores is arbitrary; only 
Table III. Demographics, disease, and treatment history ${ }^{a}$

\begin{tabular}{|c|c|c|}
\hline Characteristic & Category & $\mathrm{n}(\%)^{\mathrm{b}}$ \\
\hline \multicolumn{3}{|l|}{ Demographics $(n=284)$} \\
\hline \multirow[t]{2}{*}{ Gender } & Male & $150(52.8)$ \\
\hline & Female & $134(47.2)$ \\
\hline \multirow[t]{5}{*}{ Marital status } & Married & $140(49.3)$ \\
\hline & Widowed & $7(2.5)$ \\
\hline & Divorced or separated & $45(15.8)$ \\
\hline & Single & $74(26.1)$ \\
\hline & Other & $18(6.3)$ \\
\hline \multicolumn{2}{|l|}{ Age, y [mean (SD)] } & $41.6(11.9)$ \\
\hline \multirow[t]{5}{*}{ Highest level of education } & Secondary school qualification or less & $129(45.4)$ \\
\hline & University first degree & $86(30.3)$ \\
\hline & Some postgraduate education but no higher degree obtained & $22(7.7)$ \\
\hline & Postgraduate or professional qualification & $42(14.8)$ \\
\hline & Other & $5(1.8)$ \\
\hline \multirow[t]{4}{*}{ Employment } & Employed (full-time/part-time/self) & $178(62.7)$ \\
\hline & Student/retired/other & $38(13.4)$ \\
\hline & Unable to work & $41(14.4)$ \\
\hline & Unemployed & $27(9.5)$ \\
\hline \multicolumn{3}{|l|}{ Disease and treatment history $(n=226)^{c}$} \\
\hline \multirow{5}{*}{$\begin{array}{l}\text { How long ago were you diagnosed with } \\
\text { hepatitis } C \text { ? }\end{array}$} & Less than 6 mo & $16(7.1)$ \\
\hline & $6 \mathrm{mo}$ to $1 \mathrm{y}$ & $34(15.0)$ \\
\hline & $2-5 y$ & $67(29.7)$ \\
\hline & $6-10 y$ & $46(20.4)$ \\
\hline & More than $10 y$ & $63(27.9)$ \\
\hline \multirow[t]{4}{*}{ Treatment experience summary } & Treatment naïve & $61(27.0)$ \\
\hline & On treatment for the first time & $47(20.8)$ \\
\hline & Not currently on treatment but treated in the past & $74(32.7)$ \\
\hline & Currently on treatment and treated in the past & $44(19.5)$ \\
\hline \multirow{11}{*}{$\begin{array}{l}\text { Why didn't you start treatment for your } \\
\text { hepatitis } C \text { right away? }\end{array}$} & I didn't realize that hepatitis $C$ was serious & $13(19.7)$ \\
\hline & My doctor said I didn't need treatment because my liver was okay & $13(19.7)$ \\
\hline & I was worried about the side effects & $15(22.7)$ \\
\hline & I didn't think the medicine would work for me & $5(7.6)$ \\
\hline & I thought better treatment might be available in the future & $8(12.1)$ \\
\hline & The medicines were too expensive & $5(7.6)$ \\
\hline & I didn't want my friends, loved ones, or co-workers to know I had hepatitis C & $6(9.1)$ \\
\hline & $\begin{array}{l}\text { I was afraid that the medicines for hepatitis } \mathrm{C} \text { would interact with medicines I was } \\
\text { taking for another condition }\end{array}$ & $6(9.1)$ \\
\hline & My doctor said that treatments for hepatitis $C$ were not a good idea for people like me & $1(1.5)$ \\
\hline & I couldn't fit the treatment into my life at the time & $9(13.6)$ \\
\hline & Other & $16(24.2)$ \\
\hline \multicolumn{3}{|c|}{ a Proportions may not sum to 100 due to rounding. } \\
\hline \multicolumn{3}{|l|}{ b Unless otherwise stated. } \\
\hline \multicolumn{3}{|c|}{ c Participants in Spain $(n=58)$ were not asked any disease or treatment history questions due to ethical restrictions. } \\
\hline \multicolumn{3}{|c|}{ d Participants could select more than one category. } \\
\hline
\end{tabular}


relative differences among attribute levels are meaningful. Given the range of levels of each attribute in the study, chance of SVR is the most important attribute for $\mathrm{HCV}$-infected patients participating in the survey, followed by chance of severe anemia and chance of severe rash. The importance of changes in treatment type and the number of times per day the third medicine is taken was not statistically significant. Even though mean relative importance differed among all attributes, these differences are not always statistically significant. For example, SVR is statistically significantly more important than the other four attributes $(\mathrm{p}<0.05)$. Also, SVR is 1.7 times as important as chance of severe anemia and 5.9 times as important as chance of severe rash $(\mathrm{p}<0.05)$.

\section{Discussion}

While the rate of new HCV infection in the US and Europe declined dramatically following the development and implantation of blood screening in $1992,{ }^{[3]}$ the burden of chronic HCV infection is expected to increase through the year 2020 and beyond as the patient population ages and their disease progresses. ${ }^{[27-30]}$ In the absence of new treatments that would reverse liver damage, the primary intervention that could effectively reduce the health impact of HCV infection in the US is antiviral therapy. Davis et al. ${ }^{[28]}$ estimated that the risk of liver-related death could be reduced by $36 \%$ with current peginterferon-based therapy, but only if all infected patients were treated.

Since a universal treatment strategy is not feasible, the primary hope for reducing the burden of $\mathrm{HCV}$ and its complications lies in improving the effectiveness of therapy. However, even highly effective therapies will be unsuccessful if patients are not willing to take them. Treatment providers must understand patients' subjective assessments of the benefits, risks, and burden of therapy if newly emerging treatments such as protease inhibitors and

Table IV. Regression results

\begin{tabular}{|c|c|c|c|c|c|c|c|c|}
\hline \multirow[t]{2}{*}{ Variable $^{\mathrm{a}, \mathrm{b}}$} & \multicolumn{4}{|c|}{$\begin{array}{l}\text { Mean estimates: random parameters in utility } \\
\text { function }\end{array}$} & \multicolumn{4}{|c|}{ SDs of parameter distributions } \\
\hline & Coefficient & SE & $\mathrm{t}$-Statistic & p-Value & Coefficient & SE & t-Statistic & $p$-Value ${ }^{c}$ \\
\hline Treatment $A$ & 0.03 & 0.03 & 0.78 & 0.43 & 0.07 & 0.04 & 1.84 & 0.07 \\
\hline Treatment B & 0.02 & 0.03 & 0.56 & 0.58 & 0.07 & 0.04 & 1.62 & 0.10 \\
\hline Treatment C & 0.09 & 0.12 & 0.70 & 0.48 & 0.18 & 0.17 & 1.07 & 0.29 \\
\hline Treatment D & 0.00 & 0.03 & -0.03 & 0.98 & 0.01 & 0.04 & 0.17 & 0.87 \\
\hline Treatment E & -0.05 & 0.09 & -0.53 & 0.60 & -0.14 & 0.19 & -0.74 & 0.46 \\
\hline $85 \%$ chance of getting rid of virus completely & 0.92 & 0.05 & 18.15 & 0.00 & 0.98 & 0.05 & 19.02 & 0.00 \\
\hline $70 \%$ chance of getting rid of virus completely & 0.15 & 0.03 & 5.22 & 0.00 & 0.09 & 0.06 & 1.53 & 0.13 \\
\hline $45 \%$ chance of getting rid of virus completely & -1.07 & 0.05 & -20.18 & 0.00 & -1.07 & 0.08 & -13.45 & 0.00 \\
\hline No chance of severe rash & 0.19 & 0.03 & 5.89 & 0.00 & 0.11 & 0.04 & 2.62 & 0.01 \\
\hline $5 \%$ chance of severe rash & -0.05 & 0.03 & -1.47 & 0.14 & 0.02 & 0.04 & 0.45 & 0.66 \\
\hline $12 \%$ chance of severe rash & -0.14 & 0.03 & -4.50 & 0.00 & -0.13 & 0.06 & -1.99 & 0.05 \\
\hline No chance of severe anemia & 0.60 & 0.05 & 12.05 & 0.00 & 0.73 & 0.06 & 12.14 & 0.00 \\
\hline $20 \%$ chance of severe anemia & 0.00 & 0.03 & 0.01 & 0.99 & 0.07 & 0.06 & 1.23 & 0.22 \\
\hline $70 \%$ chance of severe anemia & -0.60 & 0.05 & -11.99 & 0.00 & -0.81 & 0.09 & -8.81 & 0.00 \\
\hline 3 tablets every 12 hours & 0.01 & 0.03 & 0.44 & 0.66 & 0.03 & 0.05 & 0.52 & 0.60 \\
\hline 2 tablets every 8 hours & 0.02 & 0.03 & 0.49 & 0.62 & 0.01 & 0.05 & 0.23 & 0.82 \\
\hline 4 tablets 3 times a day & -0.03 & 0.03 & -0.93 & 0.35 & -0.04 & 0.07 & -0.53 & 0.60 \\
\hline \multicolumn{9}{|c|}{$\begin{array}{l}\text { The omitted category is the worst level. The categorical variables were effects coded }(1,0,-1) \text {, which means that the omitted category was } \\
\text { the negative sum of the other variables. All parameters were Normally distributed. }\end{array}$} \\
\hline \multicolumn{9}{|l|}{ b See table II for definitions of treatments A-E. } \\
\hline \multicolumn{9}{|c|}{ c p-Values below 0.05 indicate heterogeneous preferences for the attribute level. } \\
\hline $\mathbf{S D}=$ standard deviation; $\mathbf{S E}=$ standard error. & & & & & & & & \\
\hline
\end{tabular}




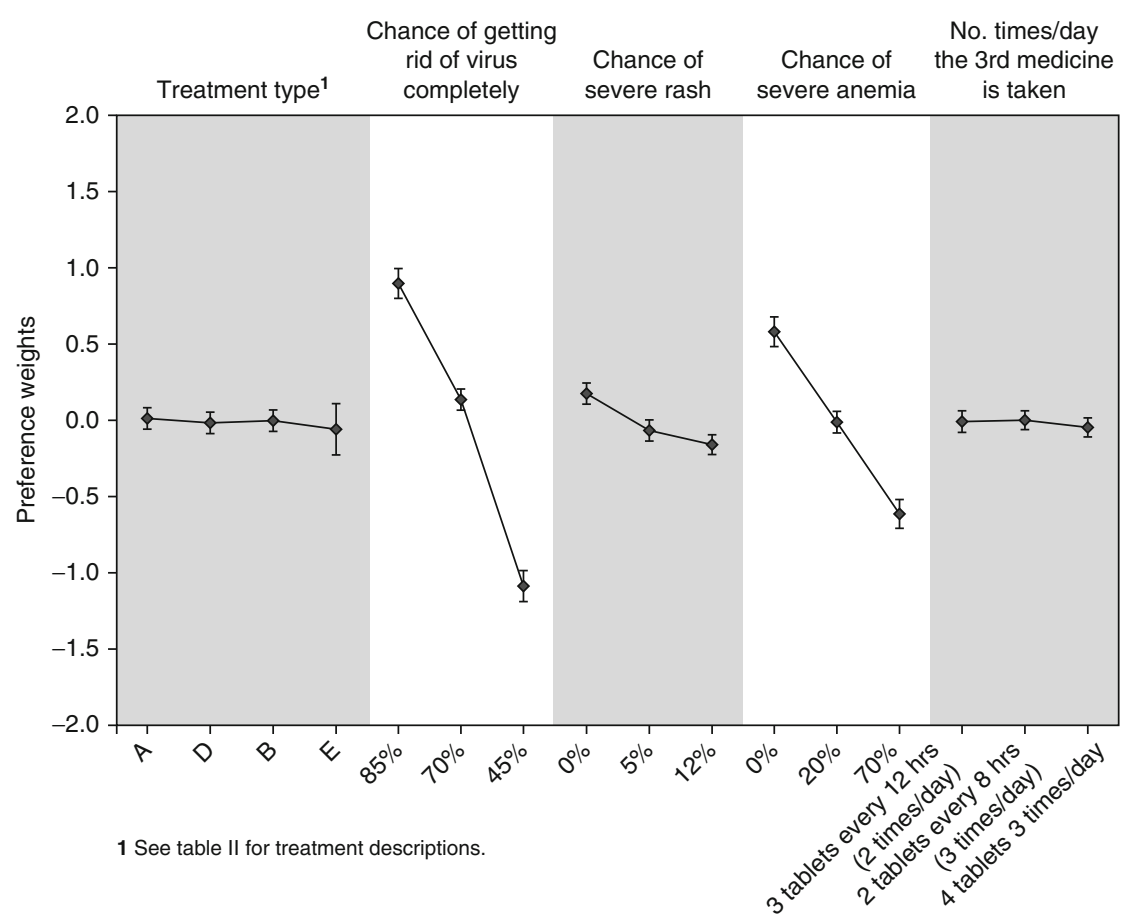

Fig. 2. Treatment attribute preference weights $(n=276)$. The vertical bars denote the $95 \%$ confidence interval around the point estimate.

other DAAs are to measurably impact projected trends in HCV-related liver disease. Yet, existing evidence suggests that providers underestimate the relative importance of side effects and other aspects of HCV treatment to patients. ${ }^{[7,31,32]}$ To our knowledge, this is the first quantitative assessment of patients' preferences for the likely features of what may soon become the dominant treatment for chronic HCV infection: peginterferon plus ribavirin plus a DAA.

The results from this survey provide important information that may guide decision making and promote patient-centered care. ${ }^{[33]}$ First, while the patients in our survey are concerned about additional side effects associated with the addition of DAAs to treatment, they are willing to accept these risks in exchange for improvements in the likelihood of successful treatment. Second, the absence of a ranking in preferences across the four hypothetical therapies, controlling for standard of care, suggests that patients are less concerned with duration per se than they are with other aspects of treatment. That is, patients are willing to accept additional weeks of therapy for a sufficient improvement in treatment effectiveness. Conversely, reductions in treatment duration alone may not induce patients to begin therapy, presenting a challenge to improving HCV treatment rates.

One reason for the relative unimportance of treatment duration in this study may be the relatively high importance of SVR. Fraenkel and colleagues $^{[12]}$ found that HCV-infected patients who perceived higher benefits of treatment were much more willing to accept treatment-related side effects. Similarly, Hauber and colleagues ${ }^{[11]}$ found that increases in the rate of SVR were more than twice as important to patients as avoiding days with flu-like symptoms. There also is evidence that achieving SVR has a significant and independent impact on patients' health-related quality of life during treatment. ${ }^{[34]}$ 
A second possible reason why participants in this study may be relatively unconcerned about reductions in treatment duration is 'duration neglect. ${ }^{[35-38]}$ Duration neglect is a phenomenon observed in empirical research where people tend to ignore or under-weight duration when making decisions. In related research, researchers have identified an additive duration effect in which people appear to care about duration, but the effect of duration on decision making is weak and does not depend on the intensity of the experience over a given duration. ${ }^{[39]}$ In the current study, participants appeared to ignore or under-weight duration, even though longer durations are associated with the potentially troublesome side effects of standard of care. This is particularly surprising given the fact that a large majority of participants in this study were treatment experienced and that patients were reminded of the side effects associated with standard of care in the survey. However, the conclusion that patients do not value decreases in duration independently from other treatment attributes, though counter-intuitive, is consistent with empirical findings in the psychology and economics literatures.

The results presented here are best interpreted with an awareness of several issues and qualifications. First, while choice-format conjoint analysis methods are increasingly used to support postmarketing regulatory decisions, identify optimal treatment guidelines, and promote patient-centered medicine, ${ }^{[14,40,41]}$ they have limitations. The most important limitation is that participants evaluate hypothetical treatments. These trade-offs are intended to simulate possible clinical decisions but obviously do not have the clinical consequences of actual decisions. Thus, differences may arise between stated and actual choices. Empirical examination of the correspondence between stated and revealed preferences in healthcare is encouraging, but limited, to our knowledge, to a single study examining physicians' alcoholism medication prescribing decisions. ${ }^{[42]}$

In this study, an attempt was made to minimize such potential differences by offering alternatives that mimic real-world trade-offs as closely as

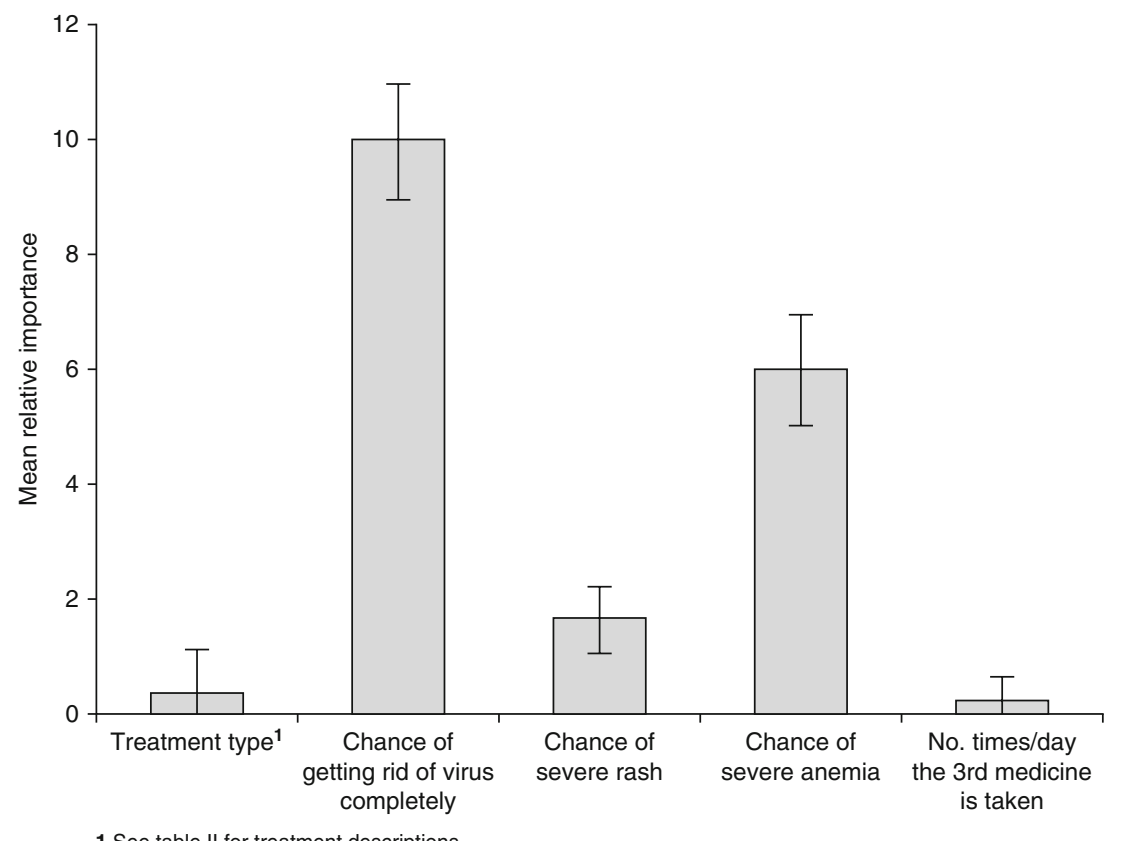

Fig. 3. Treatment attribute mean relative importance scores $(n=276)$. The vertical bars denote the $95 \%$ confidence interval around the point estimate. 
possible, with the exception that participants were presented with a forced-choice response (i.e. they had to select one of the two hypothetical treatment options presented). In pre-tests, no participants indicated that they would refuse therapy with any of the options presented. Nevertheless, there are many individual, personal factors and circumstances that can influence actual treatment decisions that are not accounted for in our study. For example, cost is sometimes cited as a barrier to treatment in the US, but not in the other surveyed countries. A number of other possible attributes were considered for inclusion in the study, including jaundice and the chance of a rapid virologic response (RVR) at week 4 . In pre-tests, patients ignored jaundice and RVR when making their choices and consistently confirmed the lack of importance of those features when queried.

Eliminating attributes that do not materially affect decision making reduces the cognitive burden on participants. If the cognitive burden is too high, respondents may employ simplifying heuristics to facilitate the task of choosing among the alternatives presented. A common strategy used to simplify choice tasks is to focus on a single attribute, whereby the respondent always chooses the alternative with the best or most attractive level of the attribute. When participants dominate on a single attribute, their preferences between attributes cannot be observed, obviating the purpose of the survey. In survey pre-testing, patients generally had strong preferences for SVR, but they were willing to trade improvements in SVR to offset treatment risks and other, negatively viewed treatment attributes. Patients were not observed having difficulty completing the choice tasks in pre-tests of the final instrument, nor was the use of any simplifying decision-making strategies detected. In the final survey, fewer than $3 \%$ of respondents dominated on an attribute, and no more than two respondents dominated on any particular attribute. The success of the pretests and the lack of dominance in the final survey are evidence that cognitive burden was not an issue in this study.

A second limitation of conjoint studies concerns the absolute values and ranges for the attribute levels in the study. Thus, the preference weight results are valid and should be interpreted only over the specific ranges of risk and other attribute levels presented in the survey. The ranges for the risk attribute levels in the present study encompassed the expected clinical prevalence but also included levels strong enough to induce trade-offs between attributes. If the levels were set too low or too high, we would never observe any trade-offs between anemia risk and the other treatment attributes. One purpose of the pre-test interviews was to inform the range and absolute values of the levels.

Random-parameters logit was used to account for unobserved heterogeneity in responses across respondents. Understanding heterogeneity in choice models is particularly difficult because the models estimate the effect of attribute levels on choice. Attribute levels change across alternatives, but individual-specific characteristics do not. Therefore, individual-specific attributes drop out of the estimator unless they are interacted with the attribute levels. Estimation of an interaction model would have required too many degrees of freedom to be supported by our sample size. Similarly, the study was not powered to conduct a splitsample analysis (i.e. by country). A third general approach to testing for heterogeneity, latent class analysis, was conducted and identified three preference groups. Continuous age was the only observed, statistically significant predictor of class. The respondents' country was not a statistically significant predictor of class. Because we found no signal to indicate that a respondent's country or any other demographic characteristic measured in the study, with the possible exception of age, explained differences in preferences, preference heterogeneity was treated as unobserved.

The relatively low prevalence of chronic $\mathrm{HCV}$ infection among the general US population poses challenges for the conduct of quantitative, patientreported preference studies. Online surveys, when conducted using established patient panels, allow access to large numbers of patients in a relatively short time period, making important information about patients' preferences and decision making available in a timely manner. Internet-based research has been used increasingly to quantify preferences for attributes of health, healthcare, and healthcare policy. ${ }^{[11,43-45]}$ 
While the demographic characteristics of participants with internet access may differ from those of individuals without such access, the psychometric properties of web-based versus paper-and-pencil surveys are comparable. ${ }^{[46]} \mathrm{A}$ full comparison of patient demographics between the overall HCVinfected populations in each of the five survey countries and the study sample was not possible, but data from the National Health and Nutrition Examination Survey (NHANES) indicate that the mean age of individuals infected with HCV in the US, including those with undiagnosed infection, is approximately 47 years, with $62 \%$ of infections occurring in males. ${ }^{[47]}$ Thus, participants in this survey were slightly younger and less likely to be male than the average $\mathrm{HCV}$-infected individual in the US.

\section{Conclusion}

Results from the present study suggest that patients with chronic HCV infection place a very high premium on SVR and are willing to accept longer treatment duration for sufficient improvement in the chance of a successful treatment outcome. With new therapies for HCV on the very near horizon, an understanding of how patients view the benefits and risks of treatment is necessary for treatment planning at both the patient and the public health levels. Additionally, patient preferences may inform the development of future HCV treatments.

\section{Acknowledgments}

The authors would like to thank Vikram Kilambi, Ryan Ziemiecki, and Lauren Donnalley of RTI Health Solutions (RTI-HS) and Jonathan Schelfhout and David R. Nelson at UF for their assistance on several study activities. This study was funded by Johnson \& Johnson (J\&J) Pharmaceutical Services, Raritan, NJ, USA through a contract with RTI-HS. DF and AA are full-time employees of J\&J, and AFM and ABH are full-time employees of RTI-HS. TLK participated in the study as an independent consultant to RTI-HS and has never received any support directly from the study sponsor.

The study was conceived by TLK, ABH, and DF and executed by TLK, ABH, and AFM. DF and AA provided important background information and served as consultants regarding survey design, patient recruitment, and interpretation of results. TLK drafted the manuscript, and all authors provided important intellectual contributions to the content. TLK, AFM, and ABH had full access to all of the data in the study, and all authors approved the final version of the manuscript. Full responsibility for all results and conclusions lies with the corresponding author, TLK.

\section{References}

1. Salomon JA, Weinstein MC, Hammitt JK, et al. Costeffectiveness of treatment for chronic hepatitis $\mathrm{C}$ infection in an evolving patient population. JAMA 2003 Jul 9; 290 (2): 228-37

2. Strader DB, Wright T, Thomas DL, et al. Diagnosis, management, and treatment of hepatitis C. Hepatology 2004 Apr; 39 (4): 1147-71

3. European Association for the Study of the Liver. EASL clinical practice guidelines: management of hepatitis $\mathrm{C}$ virus infection. J Hepatol 2011 Aug; 55 (2): 245-64

4. Butt AA, Justice AC, Skanderson M, et al. Rate and predictors of treatment prescription for hepatitis C. Gut 2007 Mar; 56 (3): 385-9

5. Kanwal F, Hoang T, Spiegel BM, et al. Predictors of treatment in patients with chronic hepatitis $\mathrm{C}$ infection: role of patient versus nonpatient factors. Hepatology 2007 Dec; 46 (6): 1741-9

6. Markowitz JS, Gutterman EM, Hodes D, et al. Factors associated with the initiation of alpha-interferon treatment in Medicaid patients diagnosed with hepatitis C. J Viral Hepat 2005 Mar; 12 (2): 176-85

7. Morrill JA, Shrestha M, Grant RW. Barriers to the treatment of hepatitis $\mathrm{C}$ : patient, provider, and system factors. J Gen Intern Med 2005 Aug; 20 (8): 754-8

8. Khokhar OS, Lewis JH. Reasons why patients infected with chronic hepatitis $\mathrm{C}$ virus choose to defer treatment: do they alter their decision with time? Dig Dis Sci 2007 May; 52 (5): 1168-76

9. Doab A, Treloar C, Dore GJ. Knowledge and attitudes about treatment for hepatitis $\mathrm{C}$ virus infection and barriers to treatment among current injection drug users in Australia. Clin Infect Dis 2005 Apr 15; 40 Suppl. 5: S313-20

10. Nelson DR. Hepatitis C drug development at a crossroads. Hepatology 2009 Oct; 50 (4): 997-9

11. Hauber AB, Mohamed AF, Beam C, et al. Patient preferences and assessment of likely adherence to hepatitis $\mathrm{C}$ virus treatment. J Viral Hepatitis 2011; 18 (9): 619-27

12. Fraenkel L, Chodkowski D, Lim J, et al. Patients' preferences for treatment of hepatitis C. Med Decis Making 2010 Jan; 30 (1): 45-57

13. Hensher DA, Rose JM, Greene WH. Applied choice analysis. Cambridge: Cambridge University Press, 2005

14. Ryan M, Farrar S. Using conjoint analysis to elicit preferences for health care. BMJ 2000 Jun 3; 320 (7248): 1530-3

15. McFaddden D. Conditional logit analysis of qualitative choice behavior. In: Zarembka P, editor. Frontiers in econometrics. New York: Academic Press, 1974: 105-42

16. Thurstone LL. A law of comparative judgment. Psychol Rev 1994; 101 (2): 266-70

17. Ryan M, Scott DA, Reeves C, et al. Eliciting public preferences for healthcare: a systematic review of techniques. Health Technol Assess 2001; 5 (5): 1-186

18. Dey A. Orthogonal fractional factorial designs. New York: Halstead Press, 1985 
19. Huber J, Zwerina K. The importance of utility balance in efficient choice designs. J Marketing Res 1996; 33: 307-17

20. Kanninen B. Optimal design for multinomial choice experiments. J Marketing Res 2002; 39: 214-27

21. Lipkus IM, Hollands JG. The visual communication of risk. J Natl Cancer Inst Monogr 1999; 25: 149-63

22. Johnson FR, Ozdemir S, Mansfield C, et al. Crohn's disease patients' risk-benefit preferences: serious adverse event risks versus treatment efficacy. Gastroenterology 2007 Sep; 133 (3): 769-79

23. Train K. Discrete choice methods with simulation. Cambridge: Cambridge University Press, 2003

24. Train K, Sonnier G. Mixed logit with bounded distributions of correlated partworths. In: Scarpa R, Alberini A, editors. Applications of simulation methods in environmental and resource economics. Dordrecht: Springer Publisher, 2005: 117-34

25. Johnson FR, Hauber B, Ozdemir S, et al. Are gastroenterologists less tolerant of treatment risks than patients? Benefit-risk preferences in Crohn's disease management. J Manag Care Pharm 2010 Oct; 16 (8): 616-28

26. Nahuelhual L, Loureiro ML, Loomis J. Using random parameters to account for heterogenous preferences in contingent valuation of public open spaces. J Agr Resource Econ 2004; 29 (3): 537-52

27. Davis GL, Albright JE, Cook SF, et al. Projecting future complications of chronic hepatitis $\mathrm{C}$ in the United States. Liver Transpl 2003 Apr; 9 (4): 331-8

28. Davis GL, Alter MJ, El-Serag H, et al. Aging of hepatitis C virus (HCV)-infected persons in the United States: a multiple cohort model of HCV prevalence and disease progression. Gastroenterology 2010 Feb; 138 (2): 513-21

29. Davis GL, Roberts WL. The healthcare burden imposed by liver disease in aging baby boomers. Curr Gastroenterol Rep 2010 Feb; 12 (1): 1-6

30. Wong JB, McQuillan GM, McHutchison JG, et al. Estimating future hepatitis C morbidity, mortality, and costs in the United States. Am J Public Health 2000 Oct; 90 (10): 1562-9

31. Cotler SJ, Patil R, McNutt RA, et al. Patients' values for health states associated with hepatitis $\mathrm{C}$ and physicians' estimates of those values. Am J Gastroenterol 2001 Sep; 96 (9): 2730-6

32. Schackman BR, Teixeira PA, Weitzman G, et al. Quality-of-life tradeoffs for hepatitis $\mathrm{C}$ treatment: do patients and providers agree? Med Decis Making 2008 Mar; 28 (2): 233-42

33. Bridges JF, Jones C. Patient-based health technology assessment: a vision of the future. Int $\mathbf{J}$ Technol Assess Health Care 2007; 23 (1): 30-5

34. Hassanein T, Cooksley G, Sulkowski M, et al. The impact of peginterferon alfa-2a plus ribavirin combination therapy on health-related quality of life in chronic hepatitis $\mathrm{C}$. J Hepatol 2004 Apr; 40 (4): 675-81
35. Fredrickson BL, Kahneman D. Duration neglect in retrospective evaluations of affective episodes. J Pers Soc Psychol 1993; 65: 44-55

36. Kahneman D, Fredrickson BL, Schreiber CA, et al. When more pain is preferred to less: adding a better end. Psychol Sci 1993; 4: 401-5

37. Redelmeier DA, Kahneman D. Patients' memories of painful medical treatments: real-time and retrospective evaluations of two minimally invasive procedures. Pain $1996 \mathrm{Jul}$; 66 (1): $3-8$

38. Varey C, Kahneman D. Experiences extended across time: Evaluation of moments and episodes. J Behav Decis Making 1992; 5: 169-95

39. Schreiber CA, Kahneman D. Determinants of the remembered utility of aversive sounds. J Exp Psychol Gen 2000 Mar; 129 (1): 27-42

40. Bridges JF, Hauber AB, Marshall D, et al. Conjoint analysis applications in health - a checklist: a report of the ISPOR Good Research Practices for Conjoint Analysis Task Force. Value Health 2011 Jun; 14 (4): 403-13

41. Bridges JF, Kinter ET, Kidane L, et al. Things are looking up since we started listening to patients: trends in the application of conjoint analysis in health 1982-2007. Patient 2008; 1 (4): 273-82

42. Mark TL, Swait J. Using stated preference and revealed preference modeling to evaluate prescribing decisions. Health Econ 2004 Jun; 13 (6): 563-73

43. Chang J, Kauf TL, Mahajan S, et al. Impact of disease severity and gastrointestinal side effects on the health state preferences of patients with osteoarthritis. Arthritis Rheum 2005 Aug; 52 (8): 2366-75

44. Hauber AB, Mohamed AF, Johnson FR, et al. Treatment preferences and medication adherence of people with type 2 diabetes using oral glucose-lowering agents. Diabet Med 2009 Apr; 26 (4): 416-24

45. Mohamed AF, Epstein JD, Li-McLeod JM. Patient and parent preferences for haemophilia A treatments. Haemophilia 2011 Mar; 17 (2): 209-14

46. Buchanan T, Smith JL. Using the internet for psychological research: personality testing on the World Wide Web. Br J Psychol 1999 Feb; 90 (Pt 1): 125-44

47. Centers for Disease Control and Prevention. National Health and Nutrition Examination Survey [online]. Available from URL: http://www.cdc.gov/nchs/nhanes.htm [Accessed 2010 Dec 12]

Correspondence: Dr Teresa L. Kauf, Associate Professor, Department of Pharmaceutical Outcomes and Policy, University of Florida, PO Box 100496, Gainesville, FL 326100496, USA.

E-mail: tkauf@ufl.edu 